\title{
Nomifensine: effect in Parkinsonian patients not receiving levodopa
}

\author{
DAVID M PARK, LESLIE J FINDLEY*, GEOFFREY HANKS $\dagger$, AND \\ MARTIN SANDLER ‡ \\ From the Department of Neurology, Charing Cross Hospital, London, and Southend Hospital, \\ Westcliff-on-Sea, Essex
}

SUMmaRY A clinical trial using the anti-depressant drug nomifensine in Parkinsonism is reported. Eighteen subjects not receiving levodopa participated. The drug had a small, but significant anti ${ }^{-}$ Parkinsonian effect. No troublesome side effects were encountered. In the treatment of Parkinsonism, nomifensine may be considered as an alternative to amantadine or anticholinergies, especially where depression is an added feature.

In a previous study ${ }^{1}$ the tetrahydroisoquinoline antidepressant, nomifensine, was shown to be an effective addition to treatment in patients with Parkinson's disease. This agent had previously been demonstrated in animals to facilitate both dopaminergic and noradrenergic transmission in the central nervous system. ${ }^{2-4}$ It has been suggested, on the basis of animal studies that for optimal antiParkinson effect a treatment should augment both dopaminergic and noradrenergic transmission. ${ }^{5}$ In the earlier study by Teychenne and his colleagues, ${ }^{1}$ the majority of patients were concomitantly receiving levodopa and this made it difficult to assess the relative anti-Parkinson efficacy of nomifensine. We wished to define more clearly the potency of nomifensine used alone as a treatment for Parkinson's disease and so in the present study we included only those patients who had not previously received levodopa or bromocriptine.

\section{Patients and methods}

Patients with idiopathic parkinsonism of either sex and any age were eligible for entry to the study. Only patients who had not previously received levodopa or bromocriptine were selected and all patients gave informed

\footnotetext{
- Present address: St. Mary's Hospital, Paddington, London W2 1NY. $\dagger$ Present address: Churchill Hospital, Oxford OX3 7LJ.

‡ Present address: St. Vincents Hospital, Sydney, Australia

Address for reprint requests: Dr DM Park, Southend Hospital, Prit tlewell Chase, Westcliff-on-Sea, Essex, SS0 ORY.

Accepted 6 November 1980
}

consent before entering the trial. The study took the form of a double-blind placebo-controlled crossover comparison. Patients received either active drug or placebo for a period of six weeks continuously, after which the treatment was reversed for a further six weeks. Nomifensine and placebo were presented in capsules matched for size, shape and colour and the dose unit of nomifensine was $25 \mathrm{mg}$. The daily dose of nomifensine was calculated on the basis of $200 \mathrm{mg}$ for an individual of 70 kilograms or above, and $100 \mathrm{mg}$ for subjects less than 55 kilograms, with grades in between. During the first week of each phase the patient was asked to increase the dose up to that prescribed.

Clinical evaluation The method for evaluation of physical signs and functional disability was that used previously, ${ }^{1}$ scores ranging from 0 (representing normal) to 4 (maximum deficit). Timed tests were employed for walking, writing, repetitively opposing the thumb to the fingers in each hand and alternate full finger extension and flexion in each hand.

Assessments were made before entry to the trial and at two-weekly intervals during it, so that three assessments were made during each treatment phase. The same investigator was responsible for all of the assessments of any particular patient. At each assessment a set of specified questions was used to elicit possible side effects. This included questions relating to mood, drowsiness and a disturbance of autonomic function.

Statistical methods The paired " $t$ " tests for comparison of means were used for the timed tests. In the analysis of clinical ratings, aggregated scores for several individual factors were grouped together and a Wilcoxon's matched pairs signed rank test applied. For example, under the general heading of akinesia the scores for speech disorder, facial expression, rising from a chair, and balance were 
considered together. The individual items of the clinical assessments were also looked at separately using the Sign Test.

\section{Results}

Twenty one patients were admitted to the study and 18 were included in the analysis of results. Two patients were omitted because of incomplete data and a third was withdrawn during the placebo phase because of severe headaches. The mean age of the group was 63 years (range 46 to 72 years) and there were 10 male and eight female patients. The mean weight of the patients was $66 \mathrm{Kg}$ (range 49 to $86 \mathrm{Kg}$ ). Six patients were receiving no medication. Of the others, nine were receiving anticholinergic agents alone, one amantadine alone, and two a combination of these drugs.

The important results of the clinical assessment are illustrated in figure which shows that several of the individual items showed significant improvement on nomifensine. Tremor and rigidity were reduced (by $13 \%, \mathrm{p}<0.02$; and by $3.4 \%, \mathrm{p}<0.01$ respectively); speech disorder (by $6 \%, \mathrm{p}<0.02$ ) and rising from a chair (by $8.5 \%, \mathrm{p}<0.04$ ) improved. Of the timed tests, finger dexterity showed no clear difference and in fact deteriorated slightly on nomifensine. Grip flexion/extension time was improved when the right hand was considered $(t=2 \cdot 44)$ and the writing time also improved $(t=2 \cdot 8)$.

The aggregated ratings showed a difference of $6.4 \%$ in favour of nomifensine, but this failed to achieve statistical significance.

Involuntary movements such as occur with treat- ment with L-dopa were not provoked in this trial. One patient experienced some postural faintness while receiving nomifensine, and one patient experienced headaches. Another complained of some drowsiness during the day, and one other patient became depressed after transfer to placebo. No adverse effects severe enough to necessitate any adjustment in dose were seen during the course of the study.

\section{Discussion}

The results illustrate that nomifensine does have a therapeutic effect in Parkinson's disease but that the benefits are generally marginal. On the other hand the drug was extremely well tolerated, and involuntary movements associated with levodopa treatment were not seen with nomifensine. Nomifensine is an established anti-depressant. ${ }^{7}$ It may be considered that the benefit observed in this study could have been due to a non-specific functional improvement as a consequence of mood enhancement. No measures of psychological variables were made in this study. However, none of the patients completing the trial appeared clinically depressed or reported mood changes. In the previous study $^{1}$ patients receiving levodopa experienced aggravation of their levodopa induced involuntary movements when nomifensine was added. This observation seems good supportive clinical evidence of specific anti-Parkinson activity.

The effect of nomifensine on dopaminergic synapses is disputed. Whilst it is clear that the drug is a potent dopamine-uptake inhibitor ${ }^{2}$ it has also

Fig Improvement in clinical scores with nomifensine

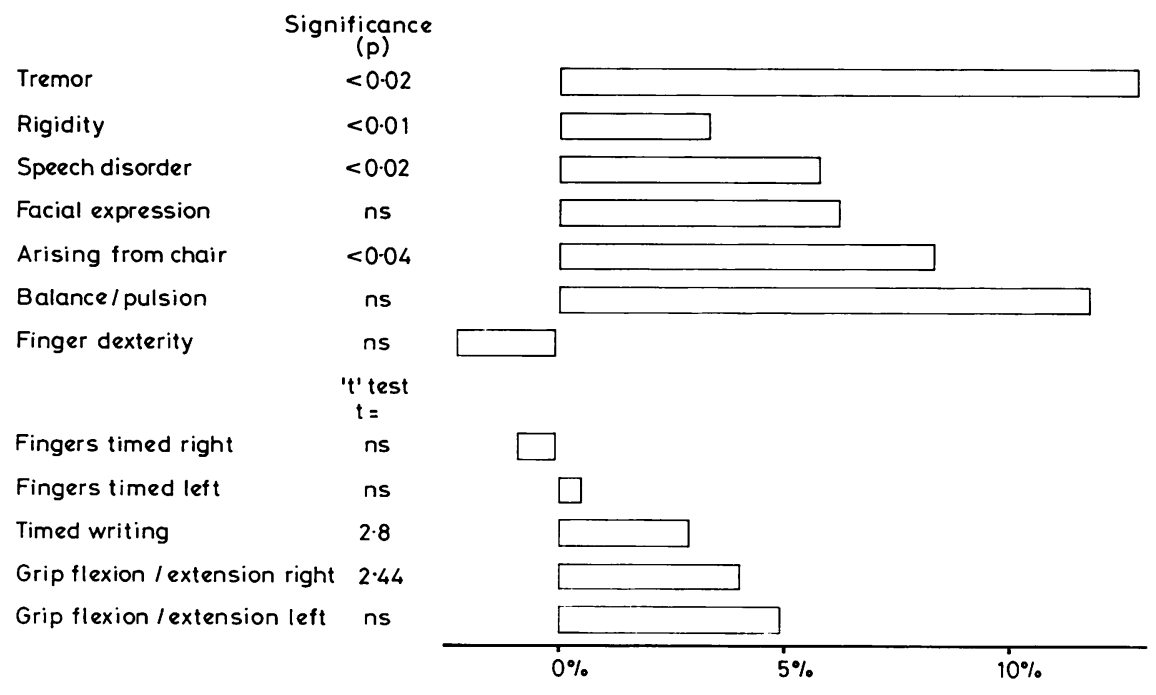


been suggested that nomifensine has post-synaptic receptor stimulating effects ${ }^{8}$ and that it may cause release of catecholamines from the pre-synaptic nerve terminal. ${ }^{9}$ A drug with merely a pre-synaptic effect is unlikely to produce marked therapeutic benefit in Parkinson's disease and the results of this trial would certainly be compatible with this pharmacological profile. A review of all the available evidence suggests that the effect of nomifensine on dopaminergic transmission is confined to blockade of reuptake. ${ }^{10}$

From a practical point of view, nomifensine may have a special role in treating Parkinsonism, complicated by depression or when depression is aggravated by levodopa or bromocriptine. Depression is frequently a part of the disability of Parkinson's disease $^{11}$ and levodopa fails to influence this mood change. ${ }^{12}$ The dose requirement of levodopa or bromocriptine may be reduced by the addition of nomifensine. ${ }^{1}$ There is also a suggestion ${ }^{13} 14$ that chronic treatment with levodopa in patients with Parkinson's disease might contribute to the earlier development of problems with involuntary movements, or to the "on-off" effect. In this respect a less aggressive form of treatment may be preferred for the patient in the early stages of the disease. Nomifensine, which is relatively free from anticholinergic and cardiovascular side effects ${ }^{15}$ could be considered as an alternative, or addition to amantadine and the anticholinergic agents.

\section{References}

1 Teychenne, PF, Park DM, Findley LJ, Rose FC, and Calne DB. Nomifensine in parkinsonism. $J$ Neurol Neurosurg Psychiatry 1976; 39:1219-21.

2 Hunt P, Kannengiesser MH, and Raynaud JP. Nomifensine: a new potent inhibitor of dopamine uptake into synaptosomes from rat brain corpus
striatum.J Clin Pharmac 1974; 26:370-1.

3 Schacht $U$ and Heptner W. Effect of nomifensine (HOE 984), a new antidepressant, on uptake of noradrenaline and serotonin and on release of noradrenaline in rat brain synaptosomes. Biochem Pharmac 1974; 23:3413-22.

4 Schacht U, Leven M, and Backer G. Studies on brain metabolism of biogenic amines. $\mathrm{Br} J$ clin Pharmac 1977; 4:77S-87S.

5 Hornykiewicz O. Mechanism of action of L-dopa in parkinsonism. In: Yahr MD ed. Advances in Neurology Vol 2, New York: Raven Press 1973:1-11.

6 Nicholson PA and Turner P ed. Proceedings of a symposium on nomifensine-January 1977. $\mathrm{Br} J$ clin Pharmac 1977; 4 Suppl 2:53S-248S.

7 Stonier PD and Jenner FA ed: Nomifensine. RSM Int Cong Sym Ser 1980; 25:1-40.

8 Costall B, Kelly DM and Naylor RJ. Nomifensine: a potent dopaminergic agonist of antiparkinson potential. Psychopharmacologia (Berl) 1975; 41 :15364.

9 Braestrup C and Scheel-Krüger J. Methylphenidatelike effects of the new antidepressant drug nomifensine (HOE 984) Eur J Pharmacol 1976; 38:305-12.

10 Hanks GW, Park DM. Nomifensine in Parkinson's Disease. In: Clifford Rose Fed. Progress in Parkinson's Disease Research. London: Pitman Medical 1981.

11 Mindham RHS. Psychiatric aspects of Parkinson's disease. Br J Hosp Med 1974; 11 :411-4.

12 Mindham RHS, Marsden CD, and Parkes JD. Psychiatric symptoms during L-dopa therapy for Parkinson's disease and their relationship to physical disability. Psychol Med 1976; 6:25-33.

13 Marsden CD, Parkes JD. Success and problems of long term levodopa therapy in Parkinson's disease. Lancet 1977; 1:345-9.

14 Rinné, UK. Recent advances in research on parkinsonism. Acta Neurol Scand (Suppl) 1978; 57(69) 77-113.

15 Stonier PD, Wittels PY. Clinical profile of nomifensine. RSM Int Cong Sym Ser 1980; 25:131-140. 